\title{
Solving a fuzzy multi-objective products and time planning using hybrid meta-heuristic algorithm: Gas refinery case study
}

\author{
Masoud Rabbani ${ }^{a^{*}}$, Hamed Farrokhi-Asl ${ }^{\mathrm{b}}$ and Mostafa Ameli ${ }^{\mathrm{a}}$
}

${ }^{a}$ School of Industrial Engineering, College of Engineering, University of Tehran, Tehran, Iran

${ }^{b}$ School of Industrial Engineering, Iran University of Science \& Technology, Tehran, Iran

\begin{tabular}{l}
\hline C H R O N I C L E \\
\hline Article history: \\
Received March 20, 2014 \\
Received in revised format \\
November 18, 2015 \\
Accepted December 182015 \\
Available online \\
December 192015 \\
\hline Keywords: \\
Fuzzy production planning \\
Multi-objective problems \\
Metaheuristic algorithms \\
Bio-geographical based \\
optimization
\end{tabular}

\section{A B S T R A C T}

\begin{abstract}
Planning is one of the most important components of gas industry production. Most big gas companies usually look for effective planning approaches to accomplish the organizational objectives including cost and time reduction as well as enhancing quality and efficiency. For planning gas refinery production, important parameters including production time, production volume, production cost and storage need to be considered. Planning different units ought to be integrated and coordinated with other departments. This article presents an intensive arithmetic model to determine the production of gas derivatives. The proposed model of this paper is formulated as a mixed integer programming and the resulted problem is solved using NSGA-II algorithm and a hybrid method called BBO/NSGA-II. The problem is also applied for a real-world case study and the results are discussed.
\end{abstract}

(C) 2016 Growing Science Ltd. All rights reserved.

\section{Introduction}

Efficient production planning can enhance the profit of gas industry companies. This industry is categorized as one of the most profitable industries in the economics of countries which have gas reserves. Reaching to suitable quality and efficiency and reduction of production costs are the goal of producer companies which can be achieved with the intelligent strategic and tactical decisions and planning. In this study, fuzzy model for production planning is considered, which is also extensively used in the literature. Liang et al. (2011), for instance, developed a fuzzy mathematical programming method to solve aggregate production planning decision problems that involve multi products and multi time periods in a fuzzy environment. The study evaluates monetary interest of related operating cost categories and provides greater computational efficiency and flexibility by adopting triangular fuzzy numbers and piecewise linear membership functions to represent both imprecise data and fuzzy goals.

\footnotetext{
* Corresponding author Tel: +9821-88021067/ Fax: +9821-88013102

E-mail address: mrabani@ut.ac.ir (M. Rabbani)

(C) 2016 Growing Science Ltd. All rights reserved. doi: $10.5267 /$ j.uscm.2015.12.002
} 
Kogan and Khmelnitsky (1995) presented an optimal production and capacity control method to solve continuous-time aggregate production planning problems that involves various expressions of production and capacity evolution processes such as regular production, subcontracting, and capacity change rates. Elamvazuthi (2009) solved a fuzzy linear programming problem in which the parameters are fuzzy quantities with logistic membership functions. In practical production systems, related environmental coefficients and parameters are often imprecise owing to incomplete and unobtainable information. To deal with imprecision, Bitran and Yanassee (1984) presented a stochastic programming model to solve multi time period aggregate production planning problems having imprecise demand with known distribution function, which derives a distribution bound using a deterministic approximation to solve the original aggregate production planning problems in an uncertain environment.

Planning for production process across the gas sector is addressed in some cases. Using planning model with uncertain conditions is common in this field and we selected fuzzy planning in this paper, and viewed the planning level beyond the processes. Accomplishing organizational objectives, planning method for gas derivatives should determine various parameters including production process, production time, production volume, resource usage, and material handling through production process, storage approach, final product characteristics and so forth, while considering demand level and available processes and resources. One can categorize gas production processes in two groups: centralized and in-centralized. In addition, all models of process-oriented production plan implementations will fall in this classification. In this research, these categories (centralized and incentralized) were analyzed according to practical examples which are representative of two ends of the spectrum.

\subsection{Centralized production method}

In this method, production planning includes scheduling, inventory control, controlling production processes and planning for material handling. System output is a centralized and integrated plan. In this method, scheduling is recurrent such that a one-to-three-month interval is selected as the total production time and a week as the discrete term. The system output, which is the production plan, is provided on weekly basis and only the first week's details will be evaluated through daily routines. The model, after that, will compute for the next weeks' plan corresponding to the first seven days' plan, demand level and demand uncertainty parameters, and this planning is accomplished according to recurrent algorithm. These models are flexible in face of process adjustment and material planning and controlling are performed with discrete manner, in fact every substance and all reservoirs will be counted. Inventory in production time is considered before and after production inventory level. The main advantage is to address such assumption through these processes that we can assign each production process to a multi-purpose reservoir according to process input and characteristics. The question which will rise is that how many multi-purpose reservoirs one needs for planning a refinery with various and many process? Do we need to know the number of processes and decomposable compounds of multi-purpose reservoirs? Obviously, increasing the number of multi-purpose reservoirs will complicate the model and monitoring the processes and inventories will be cumbersome. Avoiding there difficulties, standardized multi-purpose reservoirs, which were constructed by chemical engineers and according to under-study refinery's process, were used, and the processes were modeled in terms of multi and special purpose reservoirs. (Edith et al. 2011; Takuji 2007; Arkadej 2006; Chunpeng \& Gang 2009). Other component of this model is using robust optimization method, main characteristics of which is to avoid uncertainty, and uncertainty will be evaluated in terms of probability. In this method, confidence coefficient is used and the optimizations algorithm aims at providing a solution with biggest confidence coefficient. Chinese scholars had used this approach. This model is linear optimization method which affords adjusting the conservativeness level of robust solution. Also this method can control robust solution's conservativeness using a parameter called robustness cost. This model is a centralized planning method because of simultaneous planning for production, inventory and refinery processes. 


\subsection{In-centralized planning method}

The problem of Production planning will be decomposed in this method and two distinct problems will be evaluated and solves, while the final solution is an integrated plan. Most refineries around the world use centralized production planning such that various parameters including costs, capacities, inventories, limitation and so forth are considered and planned for. But in this model, planning is accomplished for to sub-problems. First advantage of this method is to reduce the time required for planning because the volume of two sub-problem is less than the main (centralized) problem. By production time we mean the time interval across which the production planning system transforms inputs into outputs which includes a scheduled plan for production. In this model the production processes are classified into two categories: mixing process and decomposing process. Given that these processes cannot occur simultaneously because of special consideration of transforming row material (crude oil) or inputs into final product, one can claim this classification as general and consider a storing step between each of these processes. Materials through storing step are considered as inventory (semifinished), and they will be assigned a cost coefficient and time. In addition, if this stage does not impose time, cost or both of them on system, their coefficient will become zero, and otherwise appropriated quantity should be assigned. This classification provides us with two advantages: 1) it simplifies the system process and transforms them into two machines 2) it provides easy monitoring and adjustment capability through coding the process. Generally, maintenance condition will not be considered in these models, and only time and cost are the main criteria for planning. This cause safety, quality and etc. to enter the system in terms of unaccounted costs and time (Gang \& Timo 2000; Pintoa et al., 2000; Leirasa et al., 2010).

Aiming at time and cost reduction, two sub-problems are defined for the main problem. The final output plan monitors various limitations including operational capacity of every process in given time interval, process cost in given interval, storage costs, demand unanswered cost, cost of producing no product and etc. the model inputs are of two categories:1) cost and 2) fixed quantities. Costs include cost on every process (mixing and decomposing costs), storage costs and two punishment coefficients. In this model a coefficient is considered as punishment for inventory shortage while production (input for middle processes), in a time interval and according to the next process and demand level. The plan aims at decreasing this punishment. Another punishment is final product shortage. This punishment is determined according to the chaining demand of each interval, that the model will optimize the plan considering the demand. Other inputs include production capacity, demand level, time intervals and their durations, and they will be considered as fixed/constant quantities of model inputs. They cause the planning to be executed by considering the available resources and operational limitations, and that the final planning will not be inapplicable and beyond the production capacity and that the demands will be satisfied. Another constant will be accounted for as production safety share which define the minimum production level considering the non-producing punishment. This model includes other parameters which prevent some errors, including process implementation without raw material, to occur through computerized planning. They are out of research scope (Pintoa et al., 2000; Leirasa et al., 2010; Joly et al., 2002; Young et al., 2008; Wenkai, 2005).

Sub-problem 1 refers to inventory control and sub-problem addresses process planning. Sub-problem 1 aims at optimizing storage cost and punishment to related stage inventory. Here planning is performed independently and without considering process costs and time. Sub-problem 2 will be solved for optimizing production time, total costs of all processes, non-producing punishment, demand unanswered punishment. This sub-problem is scheduled and planned independently, too. Transforming the planning system into two subsystems (sub-problems) have many advantages other than reducing planning time. Defining sub-problems, we aim to define an optimal and integrated plan. The main advantage of this method is that the outputs are under control and model's sensitivity to change is determinable, such that one cans identity calculation and planning accuracy. 


\subsection{Method novelty}

This project presents a fuzzy model for solving multi-product, multi-time period planning problems, to the best of our knowledge, is unique. Also a case study in gas sector has been presented, through which a real production planning problem is solved using NSGA-II algorithm and a hybrid algorithm proposed in this paper and finally the results of these two algorithms are compared by standard criteria and the best solution will be selected.

\subsection{Research process}

This research method includes the following steps. At first stage, the problem is described and the mathematical model is presented. Then, the deterministic and fuzzy data about gas refinery producer is shown is some tables. Two metaheuristic algorithms (NSGA-II and BBO/NSGA-II) are applied for solving the problem and obtained results are compared with each other with respect to some comparison metrics introduced in this paper.

\section{Problem Description: Case study}

For modeling and implementing of the proposed algorithm, data which is related to a refinery in Iran is used. Data were collected in fuzzy type with Refinery's combined planning unit consultation. The case study of this paper is associated with a refinery of gas network, which should add an amount to its output (in $\mathrm{m}^{3}$ ) in every interval and according to the signed contracts and its capacity. The studied refinery is part of the gas network. There is no financial penalties for unsupported demands of customers, but this will be accounted as a negative point for the management. In fact, we can define the problem as follow: a refinery is obliged to predict demand of the gas network (Employer), based on determined fuzzy demand according to usage variations and using previous data. Refinery's objective is to reduce the costs and back order and the problem is defined in particular form of Lost Sale, such that there would be no monetary or comparative punishment. There is no cost or shortage. Un-answered demand will cause a negative point to the management because gas network has the required reliability, and will cover the demand with its subsets. Also, four products which estimate the demand as a member of a network were evaluated as part of this problem, which are as follows,

\section{NG (Town)}

2. $\mathrm{CNG}$

3. LNG

4. LPG

With regarding to above mentioned facts about the refinery company, the problem is modeled aiming to minimize cost and to minimize demand deferred order level.

\subsection{Model Assumptions}

- The function has two objectives:

- Demand and the symbols of mathematical limitations are considered Fuzzy

- Equilibrium is considered for inventory.

- Back order is not allowed, and in case of unsatisfying gas network, the punishment will be a cost. In addition, we want to minimize this managerial limitation.

- Outsourcing is performed in terms of gas import, and imports are limited to a level in every interval.

- Labor and its limitations are considered.

- Overtime are limited.

- An amount was considered as maximum storage capacity of interval.

- The last interval of planning horizon should be supplied

- Non negative limitation for decision variables. 


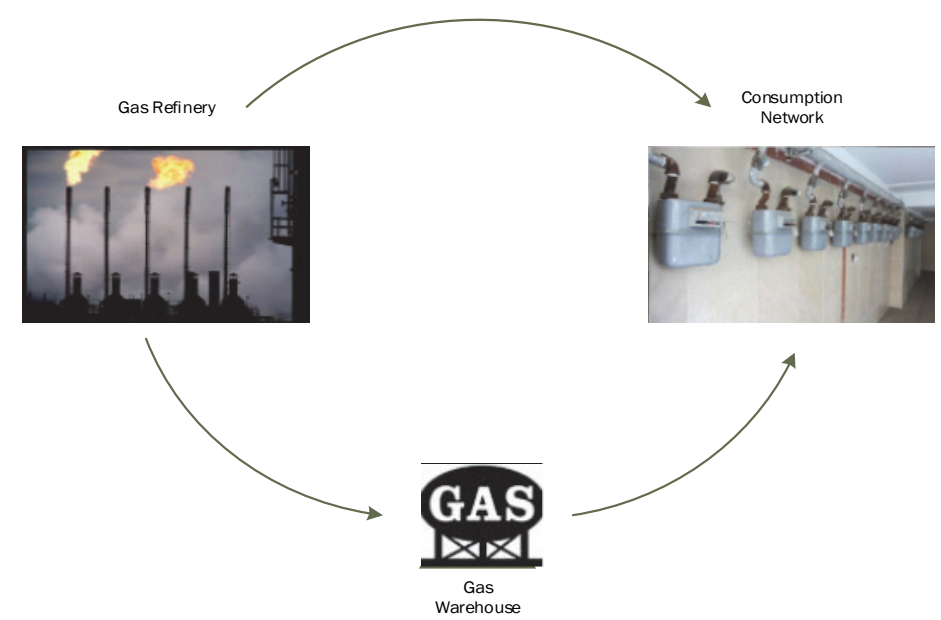

Fig. 1. Relationships of problem

\subsection{Model Description}

We proposed the following mathematical model for this Problem:

Sets:

$$
\begin{array}{ll}
T=\{0,1,2, \ldots, 12\} & \text { Set of Time period } \\
I=\{1,2,3,4\} & \text { Set of productions }
\end{array}
$$

\section{Parameters:}

$d_{i t} \quad$ Demand for product unit $i$ in time period $t$

$C 1_{i} \quad$ Production and handling cost of per product unit $i$

$C 2_{i} \quad$ Production and handling cost of per product unit $i$ in overtime

$C 3_{t} \quad$ Cost of one person/hour in working hour in time period $t$

$C 4_{t} \quad$ Cost of one person/hour at overtime in time period $t$

$C 5_{i} \quad$ Cost of supplying and handling per product unit $i$ through imports

$C 6_{i} \quad$ Cost of maintaining per product unit $i$ at reservoir

$C 7_{i} \quad$ Cost of firing an employee in time period $t$

$C 8_{i} \quad$ Cost of employing one person in time period $t$

$S_{t} \quad$ Reservoir capacity for holding the products in time period $t$

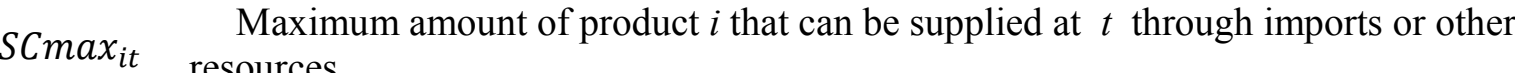

$W_{\max } \quad$ Maximum available work force at $t$.

$g \quad$ Number of work hours per worker in any $t$.

$u_{i} \quad$ Amount of required person/hour for producing product $i$ in normal work hours

$u_{i}^{\prime} \quad$ Amount of required person/hour for producing product $i$ in overtime hours

$C P_{i} \quad$ Product capacity for production $i$

Decision variables:
$x_{i t}$
Amount of product $i$ production in normal work hour in time period $t$
$Y_{i t}$
Amount of product $i$ production in overtime hour in time period $t$
$W_{t} \quad$ Required personnel in time period $t$
$H_{t} \quad$ Employed personnel in time period $t$
$L_{t} \quad$ Fired personnel in time period $t$ 
$O T_{t} \quad$ Amount of required overtime in time period $t$

$\operatorname{Inv} v_{i t} \quad$ Product $i$ inventory level at the end of time period $t$

$B_{i t} \quad$ Deferred orders of product $i$ in time period $t$

$S C_{i t} \quad$ Amount of produced product $i$ by foreign contractor in time period $t$

Objective functions:

$$
\begin{aligned}
\operatorname{Min} f_{1}= & \sum_{i=1}^{I} \sum_{t=1}^{T}\left(C 1_{i} X_{i t}+C 2_{i} Y_{i t}+C 5_{i} S C_{i t}+C 6_{i} I n v_{i t}\right) \\
& +\sum_{t=1}^{T} C 3_{t} W_{t}+C 4_{t} O T_{t}+C 7_{t} L_{t}+C 8_{t} H_{t}+\sum_{i=1}^{I} \sum_{t=1}^{T} C 5_{i} S C_{i t} \prec Z 1 \\
\min f 2= & \sum_{i=1}^{I} \sum_{t=1}^{T} B_{i t} \prec Z 2
\end{aligned}
$$

subject to

$$
\begin{array}{lc}
d_{i t}+I_{i t}-B_{i t}=I_{i, t-1}+X_{i t}+Y_{i t}+S C_{i t} & \forall i, t \\
\sum_{i=1}^{I} I_{i t} \prec S_{t} & \forall t \\
-\sum_{i=1}^{I} I_{i t} \prec-S_{t} & \forall t \\
S C_{i t} \prec S C \max _{i t} & \\
-S C_{i t} \prec-S C \max _{i t} & \forall i, t \\
W_{t} \prec W \max _{t} & \forall i, t \\
-W_{t} \prec-W \max _{t} & \forall t \\
W_{t}=W_{t-1}+H_{t}-L_{t} & \forall t \\
\sum_{i=1}^{I} u_{i}^{\prime} Y_{i t} \prec O T_{t} & \forall t \\
\sum_{i=1}^{I} u_{i}^{\prime} Y_{i t} \prec O T_{t} & \forall t \\
X_{i t}, Y_{i t}, \operatorname{Inv}_{i t}, B_{i t}, S C_{i t} & \forall i, t \\
W_{t}, H_{t}, L_{t}, O T_{t} & \forall t
\end{array}
$$

\subsection{Deterministic and Fuzzy data}

Table 1 shows the initial score of time-dependent parameters and number of personnel of each period for product development. Parameters of product type $i$ are showed in Table 2. Table 4 shows 2D parameters of product type $i$ and time periods. It should be mentioned that some of these parameters were selected according to Incorporative Planning Unit of the Refinery fuzzy and some of them as 
deterministic. Time-dependent parameters are initialized according to their definitions, thus the model is ready to be solved. Refinery's data were defined according to the problem parameters as follow:

Table 1

Variables value for $\mathrm{t}=0$

\begin{tabular}{llcc}
\hline & Unit of measure & Parameters & Value \\
\hline 1 & Man & $\mathrm{W}(0)$ & 0 \\
2 & Cubic meters & $\mathrm{I}(0)$ & 0 \\
3 & Cubic meters & $\mathrm{B}(0)$ & 0 \\
\hline
\end{tabular}

Table 2

Parameter value for $i$

\begin{tabular}{lllllll}
\hline & Unit of Measure & Parameter $/ i$ & NG(Town) & CNG & LNG & LPG \\
\hline 1 & Monetary unit & $C 1$ & 1250 & 1700 & 1700 \\
2 & Monetary unit & $C 2$ & 1280 & 1480 & 1730 & 2100 \\
3 & Monetary unit & $C 5$ & 6707 & 6973 & 9807 & 11300 \\
4 & Monetary unit & $C 6$ & 1913 & 2115 & 2802 & 2802 \\
5 & Monetary unit & $C 7$ & 100000 & 150000 & 140000 & 150000 \\
6 & Man Hours & $U$ & 0.00113 & 0.151 & 0.002 & 0.0219 \\
7 & Man Hours & $U^{\prime}$ & 0.00123 & 0.16459 & 0.00218 & 0.02387 \\
\hline
\end{tabular}

\section{Fuzzy methodology}

\subsection{Ranking fuzzy numbers}

Jiménez et al. (2007) presented fuzzy numbers ranking according to their expected interval. If we assume the input data as trapezoidal fuzzy number $\tilde{A}=\left\{a_{1}, a_{2}, a_{3}, a_{4}\right\}$, the function can be written as follows:

$$
\mu_{\tilde{A}}(x)=\left\{\begin{aligned}
0, & x \in\left(-\infty, a_{1}\right] \\
f_{A}(x), & x \in\left[a_{1}, a_{2}\right] \\
1, & x \in\left[a_{2}, a_{3}\right] \\
g_{A}(x), & x \in\left[a_{3}, a_{4}\right] \\
0, & x \in\left[a_{4}, \infty\right)
\end{aligned}\right.
$$

Insuring that the inverse of $f_{A}^{-1}(x)^{\prime}$ s and $g_{A}^{-1}(x)$ exist, we assume that $f_{A}(x)$ is continuous and ascendant and that $g_{A}(x)$ is continuous and descent. A Fuzzy number's expected interval is defined as follow:

$$
E I(\tilde{A})=\left[E_{1}^{\tilde{A}}, E_{2}^{\tilde{A}}\right]=\left[\int_{a_{1}}^{a_{2}} x d f_{A}(x),-\int_{a_{3}}^{a_{4}} x d g_{A}(x)\right]
$$

Integrating the terms and changing the variable:

$$
E I(\tilde{A})=\left[E_{1}^{\tilde{A}}, E_{2}^{\tilde{A}}\right]=\left[\int_{0}^{1} f_{A}^{-1}(\alpha) d \alpha,-\int_{0}^{1} g_{A}^{-1}(\alpha) d \alpha\right]
$$

If $f_{A}(x)$ and $g_{A}(x)$ are linear, and $\tilde{A}$ be a trapezoidal fuzzy number, then the expected interval is as follows,

$$
E I(\tilde{A})=\left[\frac{1}{2}\left(a_{1}+a_{2}\right), \frac{1}{2}\left(a_{3}+a_{4}\right)\right]
$$

Also expected value of fuzzy number $\tilde{A}$ equals expected interval divided by 2 : 


$$
E V(\tilde{A})=\frac{E_{1}^{\tilde{A}}+E_{2}^{\tilde{A}}}{2}
$$

And for trapezoidal fuzzy number $\tilde{A}$ we have:

$$
E V(\tilde{A})=\frac{a_{1}+a_{2}+a_{3}+a_{4}}{4}
$$

Definition 1: Bigger membership of two fuzzy number $\tilde{A}, \tilde{B}$ is defined as follows:

$$
\mu_{M}=(\tilde{A}, \tilde{B})=\left\{\begin{array}{c}
0 ; \text { if } E_{2}^{a}-E_{1}^{b}<0, \\
\frac{E_{2}^{A}-E_{1}^{B}}{E_{2}^{A}-E_{2}^{B}-\left(E_{1}^{A}-E_{2}^{B}\right)} ; \text { if } 0 \in\left[E_{1}^{a}-E_{2}^{b}, E_{2}^{a}-E_{1}^{b}\right] \\
1 ; \text { if } E_{1}^{a}-E_{2}^{b}>0 .
\end{array}\right.
$$

such that $\left[E_{1}^{\tilde{A}}, E_{2}^{\tilde{A}}\right],\left[E_{1}^{\tilde{B}}, E_{2}^{\tilde{B}}\right]$ are expected intervals of $\tilde{A}, \tilde{B}$, respectively. If $\mu_{M}(\tilde{A}, \tilde{B})=0.5$, then $\tilde{A}$ and $\tilde{B}$ are equal. If $\mu_{M}(\tilde{A}, \tilde{B}) \geq \alpha$ then $\tilde{A}$ is equal to and bigger than $\tilde{B}$ for at least $\alpha$, i.e. , $\tilde{A} \geq_{a} \tilde{B}$.

Definition 2: Assume that vector $x \in R^{n}$ with grade $\alpha$ would be accepted with $\min \mu_{M}(\tilde{A}, \tilde{B})=\alpha$, i.e., $\tilde{A} \geq_{a} \tilde{B}$. That

$$
\frac{E_{2}^{A x}-E_{1}^{B}}{E_{2}^{A x}-E_{1}^{A x}+E_{2}^{B}-E_{1}^{B}} \geq \alpha \Rightarrow\left[(1-\alpha) E_{2}^{A}+\alpha E_{1}^{A}\right] x \geq \alpha E_{2}^{B}-(1-\alpha) E_{1}^{B}
$$

Thus we can rearrange the fuzzy model to the absolute and exact model, considering aforementioned definitions:

$\operatorname{Min} E V(\tilde{C}) x$

s.t. $x \in\left\{x \in R^{n} \mid \tilde{A} x \geq_{\alpha} \tilde{B}, x \geq 0\right\}$

\section{Table 3}

Parameters value for each month

\begin{tabular}{|c|c|c|c|c|c|c|c|c|c|c|c|c|c|c|c|c|c|c|}
\hline \multirow[t]{2}{*}{$\#$} & \multirow[t]{2}{*}{$\begin{array}{l}\text { Paramet } \\
\text { er (UM) }\end{array}$} & \multirow{2}{*}{$\begin{array}{c}\mathrm{t} \text { (month) } \\
/ i\end{array}$} & \multicolumn{4}{|c|}{$\mathrm{NG}\left(10^{4}\right)$} & \multicolumn{4}{|c|}{$\mathrm{CNG}\left(10^{4}\right)$} & \multicolumn{4}{|c|}{$\operatorname{LNG}\left(10^{4}\right)$} & \multicolumn{4}{|c|}{$\operatorname{LPG}\left(10^{4}\right)$} \\
\hline & & & 6520 & 6540 & 6660 & 6680 & 2700 & 2850 & 3000 & 3150 & 452 & 480 & 518 & 546 & 63 & 65 & 72 & 74 \\
\hline \multirow{11}{*}{1} & \multirow{11}{*}{$\begin{array}{l}d \text { (Cubic } \\
\text { Meters) }\end{array}$} & 2 & 6520 & 6670 & 6670 & 6680 & 2850 & 2900 & 2950 & 3000 & 371 & 400 & 425 & 454 & 63 & 71 & 80 & 88 \\
\hline & & 3 & 6530 & 6680 & 6730 & 6880 & 2630 & 2780 & 2830 & 2980 & 320 & 350 & 388 & 418 & 69 & 72 & 75 & 78 \\
\hline & & 4 & 6350 & 6400 & 6400 & 6450 & 2550 & 2600 & 2600 & 2650 & 336 & 356 & 372 & 392 & 69 & 77 & 82 & 90 \\
\hline & & 5 & 6430 & 6580 & 6620 & 6770 & 2530 & 2680 & 2720 & 2870 & 321 & 340 & 365 & 384 & 51 & 58 & 59 & 66 \\
\hline & & 6 & 6690 & 6740 & 6750 & 6800 & 2890 & 2940 & 2950 & 3000 & 425 & 450 & 494 & 519 & 52 & 59 & 64 & 71 \\
\hline & & 7 & 6980 & 7000 & 7050 & 7250 & 3150 & 3300 & 3360 & 3510 & 353 & 380 & 399 & 426 & 61 & 64 & 66 & 69 \\
\hline & & 8 & 7100 & 7200 & 7300 & 7400 & 3250 & 3300 & 3300 & 3350 & 442 & 470 & 506 & 534 & 76 & 79 & 81 & 84 \\
\hline & & 9 & 7650 & 7710 & 7820 & 7880 & 3150 & 3300 & 3300 & 3450 & 448 & 470 & 510 & 532 & 68 & 75 & 77 & 84 \\
\hline & & 10 & 7890 & 7940 & 7950 & 8000 & 3350 & 3400 & 3450 & 3500 & 378 & 400 & 444 & 466 & 56 & 57 & 61 & 62 \\
\hline & & 11 & 7830 & 7890 & 7940 & 8000 & 3000 & 3150 & 3200 & 3350 & 421 & 440 & 464 & 483 & 47 & 55 & 55 & 63 \\
\hline & & 12 & 7780 & 7800 & 7880 & 7960 & 3610 & 3630 & 3750 & 3770 & 360 & 390 & 407 & 437 & 69 & 71 & 72 & 74 \\
\hline 2 & SCmax & 1 to 12 & \multicolumn{4}{|c|}{1000} & \multicolumn{4}{|c|}{0} & \multicolumn{4}{|c|}{0} & \multicolumn{4}{|c|}{0} \\
\hline 3 & $C P$ & 1 to 12 & \multicolumn{4}{|c|}{7000} & \multicolumn{4}{|c|}{3000} & \multicolumn{4}{|c|}{500} & \multicolumn{3}{|c|}{800} & \\
\hline
\end{tabular}

\begin{tabular}{cccccccccccccccc}
\hline$\#$ & $\begin{array}{c}\text { Parameter } \\
/ \mathrm{t} \text { (month) }\end{array}$ & 1 & 2 & 3 & 4 & 5 & 6 & 7 & 8 & 9 & 10 \\
\hline 1 & $C 3$ & 21889 & 21889 & 21889 & 21889 & 21889 & 21889 & 21889 & 21889 & 21889 & 21889 & 21889 & 21889 \\
2 & $C 4$ & 41667 & 41667 & 41667 & 41667 & 41667 & 41667 & 41667 & 41667 & 41667 & 41667 & 41667 & 41667 \\
3 & $C 8$ & $2 \times 10^{7}$ & $2 \times 10^{7}$ & $2 \times 10^{7}$ & $2 \times 10^{7}$ & $2 \times 10^{7}$ & $2 \times 10^{7}$ & $2 \times 10^{7}$ & $2 \times 10^{7}$ & $2 \times 10^{7}$ & $2 \times 10^{7}$ & $2 \times 10^{7}$ & $2 \times 10^{7}$ \\
4 & $C 9$ & 4166033 & 4166033 & 4166033 & 4166033 & 4166033 & 4166033 & 4166033 & 4166033 & 4166033 & 4166033 & 4166033 & 4166033 \\
5 & $S$ & $6 \times 10^{8}$ & $6 \times 10^{8}$ & $6 \times 10^{8}$ & $6 \times 10^{8}$ & $6 \times 10^{8}$ & $6 \times 10^{8}$ & $6 \times 10^{8}$ & $6 \times 10^{8}$ & $6 \times 10^{8}$ & $6 \times 10^{8}$ & $6 \times 10^{8}$ & $6 \times 10^{8}$ \\
6 & Wmax & 200 & 200 & 200 & 200 & 200 & 200 & 200 & 200 & 200 & 200 & 200 & 200 \\
\hline
\end{tabular}

Table 4

Fuzzy Demand data and Parameter value for each month 


\subsection{Biogeography-Based Optimization (BBO)}

BBO is one of the evolutional algorithms which have been proposed by Dan Simon (2008). This algorithm was used for single-object optimization of characteristic function (Ergezer \& Simon 2009; Du et al., 2009; Ma et al., 2012) and solving many real-world optimization problems such as sensor selecting problem for aircraft motor efficiency estimation (Simon 2008) and grouping satellite images. In Ma et al. (2012), island clustering property was used and islands were considered in the peninsulas. This algorithm uses non-dominated sorting and crowding distance and population diversity, and finally the results of two prominent algorithms in field of multi-objective optimization (NSGAII, AMGA) were compared according to three criteria. The results showed that this algorithm is more diverse and has better convergence. Proposed algorithm is inspired by peninsula algorithm, and aims at improving obtained solutions through consecutive generations through implementing mixed migration operator. Another effort in optimization arena with BBO algorithm was accomplished, which used BBO for multi-objective optimizing of complex systems. In Jamuna and Swarup (2012), another application of BBO for solving multi-objecting optimization was presented. The multi-objective version of BBO algorithm with Predator-Prey model was introduced in (Costa e Silva, Coelho \& Lebensztajn 2012), and remarkable results were obtained.

BBO is an evolutional, population-based algorithm, and has been inspired birds' and animals' migration among the islands. In fact, biogeography studies geographical distribution of bio species. Islands which are appropriate for settlement of geographical species have higher HSI (habitat suitability index). Factors determining HSI among other are: rainfall, flora diversity, survey properties, soil and temperature (SIVU). Islands with high HSI have more species which migrate to neighbor. Islands with high HSI have low inflow migration, because they have been occupied by other species and can admit new species. Islands with low HSI have higher inflow migration because of sparse population. Migration to such islands my increase their HSI, because appropriateness of an islands is related with its biodiversity. Employing biogeography for optimizing in the first stance had addressed to how to use natural process to solve optimization problems. As operators such as crossover and mutation have been proposed for other evolutional algorithms, including GA, Migration and mutation operators in BBO algorithm impose desired changes on generation production.

\subsubsection{Migration Operator}

Assume that we have a problem and a set of candidate solutions, which are presented by an integer vector. One can assume every integer in solution vector as a SIV. Additionally, assume that there are some methods determining the desirability level of solutions. Desirable solutions are of high HSI (habitat with high biodiversity) and the undesirables of low HSI (habitat with low biodiversity). HSI in BBO is analogue to fitness in other optimization algorithms. Every habitat (solution) of BBO have an inflow migration rate $(\lambda)$ and outflow migration rate $(\tilde{\eta})$, which are used for information sharing as a possible path to the solutions, and they are computed as follows:

$$
\begin{aligned}
& \lambda_{i}=I\left(1-\frac{k(i)}{n}\right) \\
& \mu_{i}=E\left(\frac{k(i)}{n}\right)
\end{aligned}
$$

where $I$ and $E$ are maximum possible inflow and outflow migration, respectively, and $k(i)$ presents the number of species in $i$-th habitat which is between 1 and $n$, where $n$ is the population number $n$ is for the best solution and 1 for the worst solution). Probability of every solution is modified by other solutions. When a solution is selected for modification, its inflow migration rate $(\mu)$ will be used, which specifies in probability-based manner whether or not the available SIVs should be modified. If a SIV 
of $S_{i}$ solution is selected for modification, then using outflow migration $(\tilde{\eta})$, we probabilistically specify which solution should cause a SIV, which has been selected randomly, to migrate to other $S_{i}$ solution.

\subsubsection{Mutation}

Sudden evolutions can change the HSI value of a habitat. Also they can deviate the number of species from the equilibrium (factors including unnatural material which are brought into the area from neighbor habitats or by water, diseases, natural disasters, and etc.). We model this change as SIV evolution in $\mathrm{BBO}$, and use probability of present species' number to determine the mutation rate:

$$
m(s)=m_{\max }\left(\frac{1-p_{s}}{p_{\max }}\right)
$$

where $m_{\max }$ (maximum mutation rate) is defined by the user, $P_{s}$ is the probability of that the habitat to have the species S (Simon 2008). This mutation pattern causes increase in population diversity.

\subsubsection{BBO Algorithm}

Generally, BBO algorithm is defined as follow:

Step 1. Parameter initialization: mapping problem solutions to SIVs and habitat; determining maximum number of species $S_{\text {max }}$; maximum migration rate $I, E$; maximum mutation rate $m_{\max }$; elitism parameter.

Step 2. Random creation of primarily solutions (habitats).

Step 3. Using HIS quantity, we will determine these parameters for every habitat: number of species $S$, inflow migration rate $\lambda$, outflow migration $\mu$.

Step 4. Every non elite habitat will be modified using inflow and outflow migration, and then HSI will be computed again.

Step 5. We change the probability of the number of habitat species. Then we mutate the non-elite habitat and compute the HSI quantity for every habitat again.

Step 6. In next cycle we return to step 3.

Step 7. This loop may terminate after predetermined number of generations reached or an acceptable solution to be found.

\subsection{Multi-objective Genetic Algorithm (NSGA-II)}

NSGA-II algorithm implementation steps includes:

1. First of all, a population $\left(\mathrm{P}_{0}\right)$ with $\mathrm{N}$ member is created and presented to the algorithm.

Contour $t$ used to differentiate the generations. In this stage $t=0$.

2. Implementing the crossover and mutation operators, children population $Q_{0}$ with $\mathrm{N}$ member will be created.

3. Aggregation $P_{t}$ and $Q_{t}$, a population $R_{t}$ with $2 N$ member will be created. All the population will reside in $F_{1}, \ldots, F_{k}$ using Non-dominated sorting.

4. Parent's population of $\mathrm{P}_{\mathrm{t}+1}$ will be created as follow: beginning with $\mathrm{F}_{1}$ frontier population, if $\left|P_{t+1}\right|+\left|F_{i}\right| \leq N$, then $\mathrm{F}_{\mathrm{i}}$ frontier population will be added to $\mathrm{P}_{\mathrm{t}+1}$ population. If $\left|P_{t+1}\right|+$ $\left|F_{i}\right|>N$, then some members of $F_{i}$ which has greater crowding distance will be added to $\mathrm{P}_{\mathrm{t}+1}$, that the $\mathrm{P}_{\mathrm{t}+1}$ 's population equal $N$.

5. After $P_{t+l}$ population is formed, Rank comparison will be implemented among the participants and using racing methods. A member who has a better (lower) rank will be selected. If the 
ranks are equal then the member with greater crowding distance will be selected. Then producing the children population $Q_{t+l}$ with $\mathrm{N}$ member, crossover and mutation operators will be implemented.

6. $t=t+1$ and it goes back to 3 , this cycle will continue until algorithm's stop criteria met.

\subsection{Hybrid Algorithm (BBO/NSGA-II)}

This algorithm is a meta-heuristic algorithm with BBO construct, but with the difference that in this method we used several steps of NSGA-II algorithm to improve the solve method. The differences is:

1. The conversion algorithm to a multi-objective algorithm (MOBBO)

2. Sort of population

3. Produce a new population

The steps of proposed algorithm is summarized as following:

Step 1. Producing a set of random habitat points (initial population) and sorting them by NSGA-II sorting method. The answers are the habitat points, and in fact, migration is variation of the variables. Step 2. Determining $\mu$ and $\lambda$ according to rank of solutions.

Step 3. We repeat phases 4-8 for each Habitat $i$.

Step 4. We perform phases 5-8 for every variable $k$ in habitat $i$.

Step 5. We apply the changes in $X_{i k}$ with probability of $\lambda_{\mathrm{i}}$. We perform the variations according to phases 6-8.

Step 6. Determining migration start point using $\mu$ scores randomly.

Step 7. Performing migration from $X_{j k}$ to $X_{i k}$

Step 8. Random variations (Mutations) will be applied to $X_{\mathrm{ik}}$ components.

Step 9. We evaluate the new answers.

Step 10. We combine the primary (previous) and secondary population (which resulted from migration), and create next phase's population by NSGA-II method.

Step 11. If the conditions are not met, then we should return to phase 3.

\section{Methods Comparison}

In this section first, present each criterion for each algorithm and then we calculate the criteria for two algorithm and analyze the results.

Number of Pareto solutions (NPS): This variable shows that how much shows that how much the algorithm is capable to find efficient point

Computational Time (CT): This variable shows that how much it takes for the algorithm to find the NPS

Spacing Metric (SM): This variable shows details about the uniformity of the distribution of the solutions obtained by each algorithm.

$$
S M=\sqrt{\frac{1}{N-1} \sum_{i=1}^{n}\left(d_{i}-\bar{d}\right)^{2}}
$$

where $d_{i}$ is the Euclidean distance between solutions $i$ and the closest solution which is belonged to Pareto sets of solutions. $\bar{d}$ is the average value of all $d_{i}$

Diversification Metric (DM): This variable shows the spread of solution set. 


$$
D M=\sqrt{\sum_{i=1}^{n} \max \left(\left\|x_{t}^{i}-y_{t}^{i}\right\|\right.}
$$

where $\max \left(\left\|x_{t}^{i}-y_{t}^{i}\right\|\right)$ is the Euclidean distance between the non-dominated solutions $x_{i}^{t}$ and $y_{i}^{t}$.

Space Covers (SC): This variable shows an estimation of the size of the space covered by Pareto solutions

Table 5

Comparison of two Algorithms with respect to 5 criteria

\begin{tabular}{lll}
\hline Criteria / Algorithm & Hybrid-BBO/NSGA-II & NSGA-II \\
\hline Number of Pareto Solution (NPS) & 24 & 56 \\
Computational time & 20.476913 seconds & 8.243527 seconds \\
Spacing Metric & 0.80470611 & 1.61421722541136 \\
Diversification Metric & 7861910.26 & 11777809.86 \\
Space Covers & 7736113963277.12 & 6504151207440.48 \\
\hline
\end{tabular}

Each algorithm is run in MATLAB software 20 times and the average of the numerical results in this study has been presented. According to the results, NPS obtained by NSGA-II algorithm has better performance in finding the effective points. It is also significant that this algorithm has a very low computation time. The uniformity of the proposed algorithm is better than NSGA-II according to the spacing metrics and size of the space covered by Pareto solutions is bigger in MOBBO algorithm. Obtained Pareto solutions in one of the runs is demonstrated in Fig. 3 and Fig. 4. Horizontal axis shows the first objective values and vertical axis shows the second objective value.

Table 6

Pareto solutions obtained from NSGA-II

\begin{tabular}{cccc}
\hline number of Run & number of Pareto & $z 1$ & $z 2$ \\
\hline 1 & 55 & 800268222.38 & 6977810659866.54 \\
2 & 100 & 7106382477182.57 & 849542572.08 \\
3 & 52 & 6996474016334.67 & 1000340338.90 \\
4 & 84 & 6830701378090.42 & 896755343.07 \\
5 & 63 & 6595965691025.47 & 874534938.21 \\
6 & 35 & 7217427251495.39 & 628058364.26 \\
7 & 82 & 7180708640635.27 & 705102787.07 \\
8 & 76 & 7569800457603.39 & 633071150.29 \\
9 & 70 & 7088371966304.14 & 766239434.76 \\
10 & 56 & 6935036731354.90 & 796723972.79 \\
11 & 62 & 7213240419884.16 & 807419794.61 \\
13 & 47 & 7013865527882.82 & 954059217.26 \\
14 & 45 & 6721912284863.77 & 1079329069.53 \\
\end{tabular}

Table 7

Pareto solutions obtained from Hybrid-BBO/NSGA-II

\begin{tabular}{cccc}
\hline Number of run & Number of Pareto & $z 1$ & $z 2$ \\
\hline 1 & 38 & 7683487346456.64 & 1457423358.53 \\
2 & 22 & 8056522963869.20 & 1378833408.18 \\
3 & 20 & 8261142412724.67 & 1104690696.40 \\
4 & 28 & 8141451195455.67 & 1598686991.07 \\
5 & 23 & 8119120936298.85 & 1535136530.35 \\
6 & 31 & 8014135544656.36 & 1529963895.23 \\
7 & 37 & 8429989259994.66 & 1284961290.73 \\
9 & 25 & 8523800065503.05 & 1226233053.96 \\
10 & 17 & 7589404579709.20 & 1658224263.65 \\
11 & 33 & 8184465891916.64 & 1475153416.12 \\
13 & 15 & 8605529199368.32 & 1158065936.87 \\
14 & 34 & 8339739054755.36 & 1326676924.56 \\
& 27 & 7982972822971.69 & 1474858980.26 \\
\end{tabular}




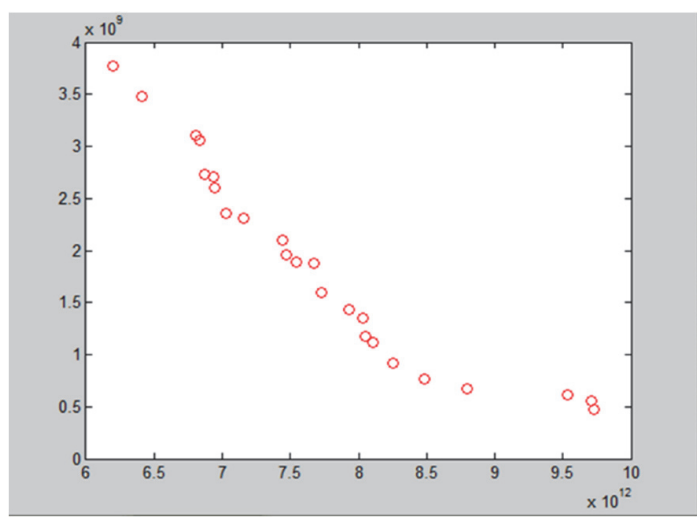

Fig. 3. Pareto solutions obtained by BBO/NSGA-II

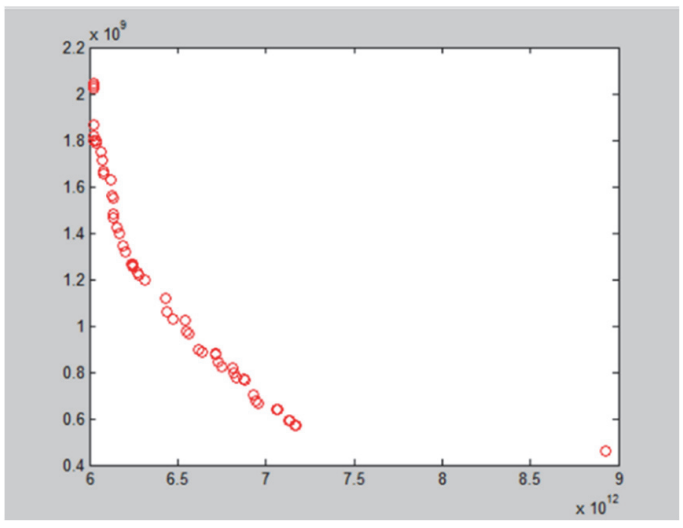

Fig. 4. Pareto solutions obtained by NSGA-II

\section{Conclusion}

This study has provided a fuzzy mathematical model for a gas refinery production planning, which could be used for the integration planning of a refinery. Also the data relating to a refinery has been used as input data. Given the complexity of the model we have created a new algorithm using the tools available to solve mathematical programming and two meta-heuristic algorithms that can obtain better results have been proposed and two solutions of the Pareto Table have been provided with NSGA-II and Hybrid-BBO/NSGA-II. The problem's data collected according to experts' opinion. Algorithm selected and improved used to study literature and analyze all available algorithms. This problem's solutions will be presented for refinery to make decisions for production scheduling based on numerical obtained results. In this study, two methods have been resolved with numbers close to the refinery and the two methods were compared. According to the comparison results, it can be said that the developed method can be very effective for this kind of problem.

According to research results, future researches can be generalized algorithm for overall mathematical programming problems. This mathematical model can be used for production planning in other industries like Oil and Petrochemical industries. Also sensitivity analysis with respect to the ideals of management can be useful.

\section{References}

Bitran, G. R., \& Yanasse, H. H. (1984). Deterministic approximations to stochastic production problems. Operations Research, 32(5), 999-1018.

Chunpeng, L., \& Gang, R. (2009). A strategy for the integration of production planning and scheduling in refineries under uncertainty. Chinese Journal of Chemical Engineering, 17(1), 113-127.

Costa e Silva, M. A., Coelho, L. D. S., \& Lebensztajn, L. (2012). Multiobjective biogeography-based optimization based on predator-prey approach. Magnetics, IEEE Transactions on, 48(2), 951-954.

Du, D., Simon, D., \& Ergezer, M. (2009, October). Biogeography-based optimization combined with evolutionary strategy and immigration refusal. InSystems, Man and Cybernetics, 2009. SMC 2009. IEEE International Conference on (pp. 997-1002). IEEE.

Ejikeme-Ugwu, E., Liu, S., \& Wang, M. (2011). Integrated refinery planning under product demand uncertainty. EN Pistikopoulos, MCG Kokossis, AC (Eds.), Computer Aided Chemical Engineering, Elsevier, 950-954.

Ejikeme-Ugwu, E., Liu, S., \& Wang, M. (2011). Integrated refinery planning under product demand uncertainty. EN Pistikopoulos, MCG Kokossis, AC (Eds.), Computer Aided Chemical Engineering, Elsevier, 950-954.

Elamvazuthi, I., Ganesan, T., Vasant, P., \& Webb, J. F. (2010). Application of a fuzzy programming technique to production planning in the textile industry. arXiv preprint arXiv:1001.2277. 
Ergezer, M., Simon, D., \& Du, D. (2009, October). Oppositional biogeography-based optimization. In Systems, Man and Cybernetics, 2009. SMC 2009. IEEE International Conference on (pp. 10091014). IEEE.

Imamura, T., Konishi, M., \& Imai, J. (2007). Method for production planning and inventory control in oil refinery. Memoirs of the faculty of engineering, Okayama University, 41, 20-30

Jamuna, K., \& Swarup, K. S. (2012). Multi-objective biogeography based optimization for optimal PMU placement. Applied Soft Computing, 12(5), 1503-1510.

Jiménez, M., Arenas, M., Bilbao, A., \& Rodrı, M. V. (2007). Linear programming with fuzzy parameters: an interactive method resolution.European Journal of Operational Research, 177(3), 1599-1609.

Joly, M., Moro, L. F. L., \& Pinto, J. M. (2002). Planning and scheduling for petroleum refineries using mathematical programming. Brazilian Journal of Chemical Engineering, 19(2), 207-228.

Kim, Y., Yun, C., Park, S. B., Park, S., \& Fan, L. T. (2008). An integrated model of supply network and production planning for multiple fuel products of multi-site refineries. Computers \& Chemical Engineering, 32(11), 2529-2535.

Kogan, K., \& Khmelnitsky, E. (1995). An optimal control method for aggregate production planning in large-scale manufacturing systems with capacity expansion and deterioration. Computers \& industrial engineering,28(4), 851-859.

Leiras, A., Hamacher, S., \& Elkamel, A. (2010). Petroleum refinery operational planning using robust optimization. Engineering Optimization,42(12), 1119-1131.

Li, W., Hui, C. W., \& Li, A. (2005). Integrating CDU, FCC and product blending models into refinery planning. Computers \& chemical engineering,29(9), 2010-2028.

Liang, T. F., Cheng, H. W., Chen, P. Y., \& Shen, K. H. (2011). Application of fuzzy sets to aggregate production planning with multiproducts and multitime periods. Fuzzy Systems, IEEE Transactions on, 19(3), 465-477.

Ma, H. P., Ruan, X. Y., \& Pan, Z. X. (2012). Handling multiple objectives with biogeography-based optimization. International Journal of Automation and Computing, 9(1), 30-36.

Pinto, J. M., Joly, M., \& Moro, L. F. L. (2000). Planning and scheduling models for refinery operations. Computers \& Chemical Engineering, 24(9), 2259-2276.

Pongsakdi, A., Rangsunvigit, P., Siemanond, K., \& Bagajewicz, M. J. (2006). Financial risk management in the planning of refinery operations.International Journal of Production Economics, 103(1), 64-86.

Simon, D. (2008). Biogeography-based optimization. Evolutionary Computation, IEEE Transactions on, 12(6), 702-713.

Liang, T. F., Cheng, H. W., Chen, P. Y., \& Shen, K. H. (2011). Application of fuzzy sets to aggregate production planning with multiproducts and multitime periods. Fuzzy Systems, IEEE Transactions on, 19(3), 465-477.

Xiong, G., \& Nyberg, T. R. (2000). Push/pull production plan and schedule used in modern refinery CIMS. Robotics and Computer-Integrated Manufacturing, 16(6), 397-410.

Kim, Y., Yun, C., Park, S. B., Park, S., \& Fan, L. T. (2008). An integrated model of supply network and production planning for multiple fuel products of multi-site refineries. Computers \& Chemical Engineering, 32(11), 2529-2535. 